\section{Fallo terapéutico por resistencia antibacteriana intra-tratamiento, a propósito de dos casos clínicos}

\author{
Luciana Robino, Nicolás Cordeiro, Virginia García-Fulgueiras, \\ Inés Bado, Gabriela Algorta y Rafael Vignoli
}

\section{Treatment failure due to acquisition of antibiotic resistance: report of two clinical cases}

Objective: We describe two cases of treatment failure due to intratreatment acquisition of antibiotic resistant microorganisms with the aim of highlighting the possible molecular mechanisms by which treatment failure occurred. Patients and Methods: We analyzed the clinical histories and the isolates obtained from 2 patients, one with a urinary tract infection (UTI) by E. coli, initially treated with cefuroxim (to which the isolate was susceptible), and another with osteoarthritis (OA) treated initially with meropenem plus vancomycin, developing $K$. pneumoniae susceptible to meropenem. During treatment, in both patients, resistant microorganisms were isolated, and empirical therapy was modified, initially with ceftriaxone and afterwards meropenem in case 1, and adding amikacin in case 2. Both strains (per patient) were compared by PFGE and resistance genes were sought by PCR. Results: Regarding the UTI, the initial strain acquired an IncFIB SHV-5-producing plasmid. In the OA case, the initial susceptible strain was substituted by a CTX-M-9 and AadB-AadA2-Aac(6')Ib-producing K. pneumoniae.

Key words: Treatment failure, antibiotic resistance, ESBL.

Palabras clave: Fallo terapéutico, resistencia antibacteriana, BLEE.

\section{Introducción}

$\mathrm{L}$ as infecciones por enterobacterias multirresistentes (definidas como aquellas que presentan resistencia a cuatro o más clases de antibacterianos ${ }^{1}$ ) son un problema creciente en el mundo ${ }^{2,3}$.

En Uruguay, en pacientes adultos hospitalizados, los antibacterianos más utilizados son los $\beta$-lactámicos ${ }^{4}$. En niños, se comunicó un incremento del consumo de aminoglucósidos y oxiiminocefalosporinas (cefuroximo, ceftriaxona y ceftazidima $)^{5}$.

El mecanismo de resistencia más frecuente a las oxiiminocefalosporinas es la inactivación enzimática mediada por $\beta$-lactamasas, fundamentalmente las $\beta$ lactamasas de espectro extendido (BLEE) ${ }^{6}$.

Este tipo de enzimas hidrolizan de modo variable las distintas oxiiminocefalosporinas, y son inhibidas por moléculas como el ácido clavulánico, sulbactam y tazobactam ${ }^{6}$. Ambas características son utilizadas para el diagnóstico de BLEE en el laboratorio clínico, tanto en sistemas manuales como automatizados ${ }^{7}$.

En Latinoamérica, recientemente se ha reportado que entre 12 y $24 \%$ de Escherichia coli y entre 50 y $60 \%$ de Klebsiella pneumoniae, aisladas a partir de pacientes internados, son productoras de $\mathrm{BLEE}^{8}$.

Por otra parte, la resistencia a aminoglucósidos está mediada principalmente por la producción de enzimas que modifican su estructura.

Universidad de la República, Montevideo, Uruguay. Facultad de Medicina, Departamento de Bacteriología y Virología, Instituto de Higiene (LR, NC, VGF, IB, GA, RV). Hospital Pediátrico, Centro Hospitalario Pereira Rossell. Montevideo, Uruguay. Laboratorio Central (GA).

Recibido: 7 de diciembre de 2012 / Aceptado: 10 de abril de 2013

Correspondencia a:

Rafael Vignoli

rvignoli@higiene.edu.uy
Dependiendo de la modificación de la molécula, estas enzimas se denominan adenilantes, acetilantes o fosforilantes de aminoglucósidos ${ }^{9}$. Los genes que codifican para dichos mecanismos frecuentemente se encuentran en elementos genéticos como integrones o trasposones, los cuales pueden encontrarse a su vez en plásmidos con la capacidad de ser transferidos entre bacterias mediante el fenómeno de conjugación ${ }^{10}$.

La elección de un agente antimicrobiano requiere considerar factores como: patrones locales de resistencia; la identificación del agente etiológico y su perfil de susceptibilidad; las características del antimicrobiano (propiedades farmacocinéticas y farmacodinámicas, capacidad bactericida, espectro de acción, concentración en diferentes parénquimas o líquidos biológicos, forma de administración e intervalos); factores dependientes del huésped (sitio de infección, prótesis, estado de inmunidad) y el entorno en donde se adquiere la infección ${ }^{11,12}$.

Existen variados reportes sobre fallas de tratamiento antimicrobiano inicial, debido a la resistencia primaria del agente infeccioso ${ }^{13,14}$. Sin embargo, existen escasas comunicaciones sobre el desarrollo de resistencia antibacteriana intra-tratamiento ${ }^{15}$.

Presentamos dos casos de fallo terapéutico en niños con infecciones por enterobacterias, asociado a cambios de susceptibilidad antimicrobiana intra-tratamiento; se caracterizaron los microorganismos y los mecanismos moleculares de resistencia.

\section{Pacientes y Métodos}

Se analizaron las historias clínicas de dos niños internados en una unidad de cuidados intensivos (UCI). Se estudiaron dos cepas de E. coli (ITU1 e ITU2) aisladas de un paciente con infección del tracto urinario (ITU) y dos cepas de K.pneumoniae (OA1 y OA2) obtenidas de líquido articular de un paciente con osteoartritis. ITU1 y OA1 fueron aisladas al ingreso e ITU2 y OA2 durante la internación.

La identificación microbiológica y de presencia de BLEE se realizó en el Laboratorio Central del Centro Hospitalario Pereira Rossell (CHPR), usando el sistema automatizado Vitek ${ }^{\circledR} 2$ (bioMérieux, Marcy l'Étoile, France). El estudio de la susceptibilidad antibacteriana se realizó mediante la técnica de Kirby-Bauer por difusión en disco y fueron interpretados siguiendo las guías EUCAST ${ }^{16}$.

La tipificación molecular se realizó mediante electroforesis de campo pulsado (EFCP $)^{17}$ en el Departamento de Bacteriología y Virología de la Facultad de Medicina, Universidad de la República. El ADN cromosómico fue digerido con $0,3 \mathrm{U} / \mathrm{mL}$ Xba I (Thermo Cientific, Lituania). La EFCP fue realizada con CHEF DR-II (Bio-Rad) a $6 \mathrm{~V} / \mathrm{cm}$ and $14^{\circ} \mathrm{C}$ con pulsos de 2 a 30 -s por $18 \mathrm{~h}$. Los fragmentos de ADN fueron visualizados mediante tinción con bromuro de etidio. La relación genética entre los aislados se realizó utilizando los criterios de Tenover ${ }^{18}$.

La presencia de genes de resistencia a oxiiminocefalosporinas, aminoglucósidos, integrones y grupos de incompatibilidad plasmídica se estudiaron con reacción de polimerasa en cadena (RPC) y secuenciación, utilizando cebadores específicos ${ }^{19,20}$.

\section{Caso 1}

Escolar de 6 años, portador de una insuficiencia renal crónica secundaria a un reflujo vesicoureteral grado 5, bilateral, con hidronefrosis y desnutrición grave. Recibía profilaxis por ITU recurrentes con cotrimoxazol y tenía el antecedente de exposición a varios antibacterianos en los últimos meses (cefuroximo, ceftriaxona y ciprofloxacina).

Ingresó a la UCI por una pielonefritis aguda e insuficiencia renal crónica reagudizada. El urocultivo realizado al ingreso fue positivo a E.coli (cepa ITU1) sensible a ampicilina, cefalotina, cefuroximo, ceftriaxona y gentamicina; resistente a ciprofloxacina y cotrimoxazol (Tabla 1). Recibió 
tratamiento con cefuroximo iv $150 \mathrm{mg} / \mathrm{kg} /$ día. Al sexto día presentó una sepsis de foco urinario. Se cambió tratamiento a ceftriaxona iv a $100 \mathrm{mg} /$ $\mathrm{kg}$ /día previa toma de urocultivo. A las $48 \mathrm{~h}$ el paciente persistía febril. El urocultivo se informó con $>10^{5} \mathrm{UFC} / \mathrm{ml}$ de $E$. coli (cepa ITU2) sensible a imipenem, meropenem, amikacina y gentamicina; resistente a ampicilina, cefalotina, cefuroximo, ceftriaxona, ciprofloxacina y cotrimoxazol (Tabla 1). Se inició meropenem iv $60 \mathrm{mg} / \mathrm{kg} / \mathrm{d}$ ía, con buena evolución clínica y urocultivo de control, intra-tratamiento, sin desarrollo bacteriano.

Tipificación molecular y mecanismo de resistencia: Mediante EFCP se confirmó que ambas cepas eran genéticamente indistinguibles (Figura 1). La cepa ITU2 presentaba una BLEE SHV-5 codificada en un plásmido conjugativo del grupo IncFIB, ausente en la cepa ITU1.

\section{Caso 2}

Recién nacido de 17 días, sin antecedentes perinatales a destacar. A los 11 días de internación en la UCI por una bronquiolitis por VRS presentó fiebre, una flebitis en un brazo por una vía venosa periférica y una tumefacción dolorosa, con rubor y calor en la segunda articulación condrocostal derecha. Con diagnóstico de osteoartritis condrocostal se inició tratamiento antibacteriano empírico con meropenem $60 \mathrm{mg} / \mathrm{kg} /$ día y vancomicina 40 $\mathrm{mg} / \mathrm{kg}$ /día durante $72 \mathrm{~h}$. Los hemocultivos fueron positivos a Klebsiella pneumoniae sensible a oxiiminocefaslosporinas, amikacina, gentamicina, meropenem e imipenem (Tabla 1). Se suspendió vancomicina y continuó con meropenem durante 14 días con negativización del hemocultivo al $7^{\circ}$ día de terapia. Mejoraron los signos locales pero continuó febril y se agregó limitación a la rotación de la cadera derecha. Con diagnóstico de probable osteoartritis coxofemoral se realizó punción articular y drenaje quirúrgico que dio salida a material purulento. En ese momento se agregó amikacina $15 \mathrm{mg} / \mathrm{kg} /$ día por el antecedente de $K$. pneumoniae aislada del hemocultivo. Del cultivo del líquido articular se obtuvo K. pneumoniae (cepa OA1) con el mismo perfil de susceptibilidad que la cepa aislada en sangre. Dado que persistió febril, se realizaron aseos quirúrgicos sucesivos aislándose, a los 17 días del primer aislado (OA1), K. pneumoniae (cepa OA2) resistente a oxiiminocefalosporinas, amikacina y gentamicina (Tabla 1). Los hemocultivos realizados durante la evolución fueron negativos, manteniéndose el mismo esquema antibacteriano. Se realizó una evaluación inmunológica que resultó normal. Luego de múltiples aseos quirúrgicos, 35 días de fiebre y 45 días de tratamiento antibacteriano se dio de alta con buena evolución posterior.

Tipificación molecular y mecanismo de resistencia: El análisis por EFCP mostró que las cepas OA1 y OA2 no estaban genéticamente relacionadas (Figura 1).

A diferencia de la cepa OA1, OA2 presentó el gen para la BLEE CTX-M-9 en un plásmido IncHI1-HI2 no conjugativo. Adicionalmente OA2 presentaba el gen $a a c\left(6^{\prime}\right) 1 b$, que confiere resistencia a amikacina y un integrón de clase 1 con los genes $a a d B$-aadA2 en la región variable, responsables de la resistencia a gentamicina.

\section{Discusión}

Las infecciones causadas por enterobacterias productoras de BLEE se asocian a mayores tasas de fallo terapéutico, mortalidad y costos hospita$\operatorname{larios}^{21}$. Los microorganismos resistentes reducen significativamente los antibacterianos disponibles para su tratamiento, ya que los genes codificantes para BLEE generalmente se asocian con otros genes de resistencia antibacteriana (aminoglucósidos, quinolonas, cotrimoxazol) ${ }^{22}$.

En el primer caso, el paciente presentó fallo al tratamiento con oxiiminocefalosporinas, dirigido a una cepa de E.coli susceptible in vitro. Esto se explicaría por la adquisición por parte de E.coli, de un plásmido conjugativo portador del gen bla $_{\mathrm{SHV}-5}$.

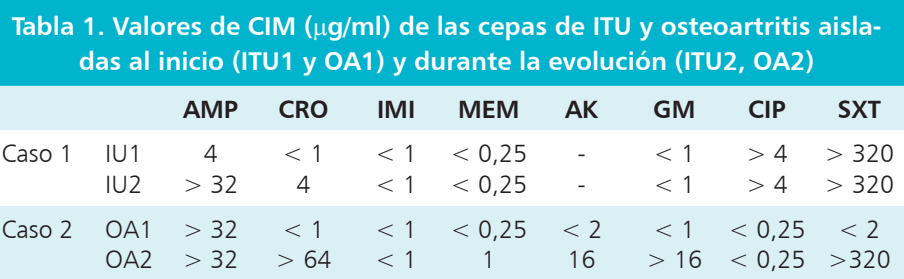

AMP: ampicilina; CRO: ceftriaxona, IMI: imipenem, MEM: meropenem, AK: amikacina, GM: gentamicina, CIP: ciprofloxacina, TMP-SMX: cotrimoxazol.

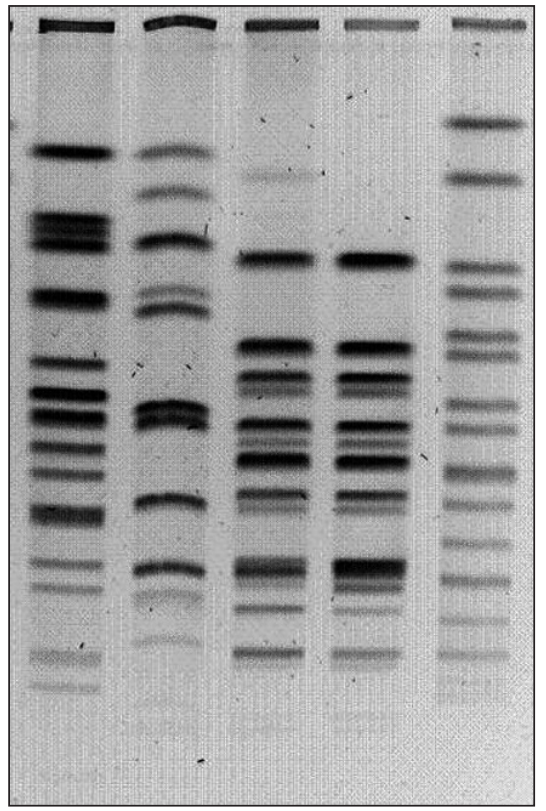

Figura 1. Electroforesis en campo pulsado. Análisis de las cepas de K.pneumoniae aisladas del paciente con osteoartritis y de E. coli aisladas del paciente con ITU. Carril 1: K. pneumoniae OA1; carril 2: K. pneumoniae OA2; carril 3: E. coli ITU1; carril 4: E. coli ITU2; carril 5: cepa de referencia Salmonella enterica ser. Braenderup H9812.

Recientemente comunicamos la detección de infecciones por enterobacterias productoras de BLEE en nuestra unidad ${ }^{20}$. Entre los factores favorecedores de BLEE están: el consumo previo de oxiiminocefalosporinas, presencia de enfermedades crónicas de base, internación en UCI en los últimos 6 meses e infección nosocomial causada por enterobacterias ${ }^{22}$. Estos factores predisponen tanto para las infecciones como para la colonización digestiva con cepas portadoras de BLEE ${ }^{2,23}$.

El primer caso presentaba como factores de riesgo una enfermedad crónica de base y exposición a múltiples antibacterianos. Dado que la cepa fue la misma constatada mediante EFCP y que la diferencia radicaba en la adquisición de un plásmido codificante de la BLEE SHV-5, se puede inferir que la co-colonización digestiva de la cepa de E. coli en estudio y otras enterobacterias productoras de BLEE habría permitido la transferencia de resistencia a la cepa aislada en orina. Lamentablemente no contamos con estudios de colonización digestiva que podrían confirmar nuestra hipótesis.

En el segundo caso, el paciente desarrolló la cepa OA1 estando en tratamiento con meropenem. Después de la adición de amikacina desarrolló una infección por otra cepa de K. pneumoniae distinta a OA1, con resistencia intermedia a aminoglucósidos y productora de BLEE (OA2), constituyendo una infección asociada a la atención de salud (IAAS).

Las enterobacterias productoras de BLEE además pueden desarrollar resistencia a carbapenémicos por disminución de la expresión de porinas ${ }^{24}$. Por otro lado, meropenem alcanza bajas concentraciones en el líquido sinovial y hueso ${ }^{25}$. Si bien ambas cepas (OA1 y OA2) eran sensibles a meropenem, en éste caso el fallo terapéutico al antibacteriano podría deberse 
a sus propiedades farmacocinéticas-farmacodinámicas y no al perfil de susceptibilidad del microorganismo. La eventual presencia de otros focos óseos secundarios, producidos durante la siembra hematógena inicial, y su diagnóstico y drenaje tardío pudieron ser causa de la lenta evolución clínica del paciente. Por otro lado, la presencia del gen $a a c\left(6^{\prime}\right)$ - $I b$ (que aumenta los niveles de resistencia a amikacina) sumado a la presencia de pus en el sitio de infección (que dificulta la acción del antibacteriano) explicarían la falla terapéutica de los aminoglucósidos. El éxito del tratamiento probablemente se debió a los múltiples aseos quirúrgicos del foco supurado mejorando la actividad del antibacteriano en dicho sitio.

Si bien el éxito terapéutico antimicrobiano depende múltiples factores describimos dos situaciones diferentes de emergencia de resistencia antibacteriana. En un caso por la adquisición de un plasmido de resistencia previamente ausente en la cepa infectante y en el otro por la sobreinfección con otra cepa con un perfil de resistencia diferente al aislado inicial.

\section{Resumen}

Se describen dos casos de fallo terapéutico en niños con infecciones por enterobacterias, asociado a cambios intra-tratamiento de la sensibilidad a antibacterianos con el objetivo de describir posibles mecanismos de falla terapéutica con estudio molecular de los agentes infecciosos detectados. Se analizaron las historias clínicas y las cepas de dos pacientes, uno con infección urinaria por E. coli inicialmente tratado con cefuroximo (al cual era sensible) y otro con osteoartritis tratado de forma empírica con meropenem-vancomicina con hemocultivos positivos a K. pneumoniae sensible a meropenem. En la evolución, ambos pacientes desarrollaron durante el tratamiento una infección por microorganismos resistentes, cambiándose a ceftriaxona y luego meropenem en el primer caso y agregando amikacina en el segundo. Se compararon las cepas por electroforesis de campo pulsado y se estudiaron mecanismos de resistencia por RPC. En el caso de ITU, la cepa inicial adquirió un plásmido IncFIB productor de la BLEE SHV-5. En el caso de OA, la cepa sensible inicial fue sustituida por otra cepa de $K$. pneumoniae productora de CTX-M-9, (AadB-AadA2), Aac(6') 1 b.

\section{Referencias bibliográficas}

1.- Macedo-Viñas M, Cordeiro N F, Bado I, Herrera-Leon S, Vola M, Robino L, et al. Surveillance of antibiotic resistance evolution and detection of class 1 and 2 integrons in human isolates of multi-resistant Salmonella typhimurium obtained in Uruguay between 1976 and 2000. Int J Infect Dis 2009; 13: 342-8.

2.- Blaschke A J, Korgenski E K, Daly J A, LaFleur B, Pavia A T, Byington C L. Extended-spectrum $\beta$-lactamase-producing pathogens in a children's hospital: A 5-year experience. Am J Infect Control 2009; 37: 435-41

3.- Qin X, Zerr DM, Weissman SJ, Englund JA, Denno DM, Klein EJ, et al. Prevalence and mechanisms of broad-spectrum beta-lactam resistance in enterobacteriaceae: a children's hospital experience. Antimicrob Agents Chemother 2008; 52: 3909-14.

4.- Cabrera A S, Sosa L, Arteta Z, Seija V, Mateos S, Perna A, et al. Rational use of antibiotics in the department of internal medicine from a university hospital: results of a pilot experience. Rev Chilena Infectol 2012; 29: 7-13.

5.- Telechea H, Speranza N, Lucas L, Santurio A, Giachetto G, Algorta G, et al. Antibiotic consumption and antimicrobial susceptibility evolution in the Centro Hospitalario Pereira Rossell in methicillin resistant Staphylococcus aureus era. Rev Chilena Infectol 2009; 26: 413-9.

6.- Paterson D L, Bonomo R A. Extended-spectrum beta-lactamases: a clinical update. Clin Microbiol Rev 2005; 18: 657-86.

7.- Treviño M, Martínez-Lamas L, Romero-Jung P, Varon C, Moldes L, Garcia-Riestra C, et al. Comparative assessment of the Vitek 2 and Phoenix systems for detection of extended-spectrum beta-lactamases. Enferm Infecc Microbiol Clin 2009; 27: 566-70.

8.- Gales A C, Castanheira M, Jones R N, Sader H S. Antimicrobial resistance among Gram-negative bacilli isolated from Latin America: results from
SENTRY Antimicrobial Surveillance Program (Latin America, 2008-2010). Diagn Microbiol Infect Dis 2012; 73: 354-60.

9.- Ramírez M S, Tolmasky M E. Aminoglycoside modifying enzymes. Drug Resist Updat 2010; 13: 151-71.

10.- Di Conza J A, Gutkind G O. Integrons: gene collectors. Rev Argent Microbiol 2010; 42: 63-78.

11.- Amsden G W, Ballow C H, Bertino J S, Kashuba A D. Pharmacokinetics and pharmacodynamics of anti-infective agents. Mandell G DR, Bennett JE, Dolin R, ed. Mandell Douglas, and Bennett's Principles and Practice of Infectious Diseases. $7^{\text {th }}$ ed. Philadelphia: Churchill Livingstone Elsevier; 2010, p. 297-308.

12.- Pillai S K, Eliopoulos G M, R C M. Principles of anti-infective therapy. Mandell G D R, Bennett J E, Dolin R, ed. Mandell Douglas, and Bennett's Principles and Practice of Infectious Diseases. $7^{\text {th }}$ ed. Philadelphia: Churchill Livingstone Elsevier; 2010, p. 267-78.

13.- Tumbarello M, Sali M, Trecarichi EM, Leone F, Rossi M, Fiori B, et al. Bloodstream infections caused by extended-spectrum-beta-lactamaseproducing Escherichia coli: risk factors for inadequate initial antimicrobial therapy. Antimicrob Agents Chemother 2008; 52: 3244-52.

14.- Tumbarello M, Spanu T, Di Bidino R, Marchetti M, Ruggeri M, Trecarichi E M, et al. The costs of bloodstream Infections caused by Escherichia coli and influence of extended-Spectrum beta-lactamase production and inadequate initial antibiotic therapy. Antimicrob Agents Chemother 2010; 54: 4085-91.

15.- Lee C H, Chu C, Liu J W, Chen Y S, Chiu C J, Su L H. Collateral damage of flomoxef therapy: in vivo development of porin deficiency and acquisition of blaDHA-1 leading to ertapenem resistance in a clinical isolate of Klebsiella pneumoniae producing CTX-M-3 and SHV-5 beta-lactamases. J Antimicrob Chemother 2007; 60: 410-3.

16.- Breakpoint tables for interpretation of MICs and zone diameters. European Committee on Anitmicrobial Susceptibility Testing EUCAST. Disponible en: http://www.eucast.org. Accedido: 14 de agosto de 2012.

17.- Vignoli R, Calvelo E, Cordeiro NF, Lucero R, Ingold E, Quintana A, et al. Association of broad-spectrum antibiotic use with faecal carriage of oxyiminocephalosporin-resistant enterobacteriaceae in an intensive care unit. J Hosp Infect 2006; 63: 306-15.

18. Tenover F, Arbeit R, Goering R, Mickelsen P A, Murray B E, Persing D H, et al. Interpreting chromosomal DNA restriction patterns produced by pulsed- field gel electrophoresis: criteria for bacterial strain typing. J Clin Microbiol 1995; 33: 2233-9.

19.- Bado I, Cordeiro N F, Robino L, García-Fulgueiras V, Seija V, Bazet C, et al. Detection of class 1 and 2 integrons, extended-spectrum $\beta$-lactamases and $q n r$ alleles in enterobacterial isolates from the digestive tract of Intensive Care Unit inpatients. Int J Antimicrob Agents 2010; 36: 453-8.

20.- García-Fulgueiras V, Bado I, Mota MI, Robino L, Cordeiro N F, Varela A et al. Extended-spectrum $\beta$-lactamases and plasmid-mediated quinolone resistance in enterobacterial clinical isolates in the paediatric hospital of Uruguay. J Antimicrob Chemother 2011; 66: 1725-9.

21.- Trecarichi E M, Cauda R, Tumbarello M. Detecting risk and predicting patient mortality in patients with extended-spectrum $\beta$-lactamase-producing Enterobacteriaceae bloodstream infections. Future Microbiol 2012; 7: 1173-89.

22.- Robino L, Telechea H, Speranza N, García-Fulgueiras V, Cordeiro N F, Bado I, et al. Risk factors for the acquisition of extended-spectrum $\beta$-lactamase-producing Enterobacteriaceae in hospitalized children. J Infect Dev Ctries 2013; 7: 361-4.

23.- Kim Y-K, Pai H, Lee H-J, Park S-E, Choi E-H, Kim J, et al. Bloodstream infections by extended-spectrum $\beta$-lactamase-producing Escherichia coli and Klebsiella pneumoniae in children: epidemiology and clinical outcome. Antimicrob Agents Chemother 2002; 46: 1481-91.

24.- Song W, Suh B, Choi J Y, Jeong S H, Jeon EH, Lee Y K, et al. In vivo selection of carbapenem-resistant Klebsiella pneumoniae by OmpK36 loss during meropenem treatment. Diagn Microbiol Infect Dis 2009; 65: 447-9.

25.- Gerding D, Hugues C, Bamberger D, J F, Larson T A. Extravascular antrimicrobial distribution and the respective blood concentrations in humans. En: Lorian V, ed. Antibiotics in Laboratory Medicine. Philadelphia: Lippincott Williams \& Wilkins; 2005, p. 719-825. 